\title{
Improving cold-chain transport system efficiency by cloud computing and wireless network
}

\author{
Zhang Rangyong ${ }^{\mathrm{a}}$, Ma Geng ${ }^{\mathrm{b}}$,Han Lingyan ${ }^{\mathrm{c}}$ \\ Shandong Provincial Key Laboratory of Computer Network, Shandong Computer Science \\ Center,(National Supercomputer Center in Jinan) Jinan 250014, China \\ azhangry@sdas.org, ${ }^{\mathrm{b}} \mathrm{mag} @$ @sdas.org, ${ }^{\mathrm{c}}$ hanlingy@sdas.org,
}

Keywords: Cold-chain transport system,Cloud computing,Wireless network.

\begin{abstract}
By using a wireless remote networking, and cloud computing platform technology, this system can collect idle space and time from every company or unit who has its isolated cold-chain transport system. Those information of every refrigerated truck's time, spare space and route can be opened to all of other customers with such demands. By this way, small quality and scattered refrigerated goods will be distributed incidentally and cheaply with those daily transport of those big company. This system greatly improve the utilization efficiency of cold chain transport system and reduce the cost of business and fragmented customers and promote the development of cold chain transport industry.
\end{abstract}

\section{Introduction}

Cold Chain transport system refers those under refrigerated and frozen food in the production, storage and transportation, sales, all aspects of the consumer will always be in front of a predetermined low temperature environment, in order to ensure food quality and reduce food losses of a systems engineering ${ }^{[1]}$. Some big refrigerated and frozen food manufactures and sales have their own team of refrigerated trucks daily transport products from one place to the other. Because of the fluctuation of sale activity, the quality of goods will be changed every day, there will be some different amount of idle space on the way. On the other hand, it is too expensive to transport some small quality refrigerated and frozen goods by one refrigerated truck on the same route.

With the rapid development of E-commerce and the technology of communication , customers can search information and products from the internet and buy almost all kind of service and product as their wish. The modern electronic technology makes people to get remote devices' running status and location easily and quickly ${ }^{[2]}$. All of those make human society t working more and more efficiently with low cost.

Install the remote digital unit to those refrigerated trucks, manufactures can know the location and the inside temperature and humidity through internet at any time, and they know its idle space and load from their work schedule with ERP system. In this case,they can put those information to cloud computing platform and sale their idle space to those potential customers without affect their own transport missions. Those potential customers can transport small quality scatted refrigerated goods cheaply and incidentally buying idle space from special refrigerated trucks’ team.

\section{System design}

This system is based on remote wireless terminal unit and cloud computing platform ${ }^{[3]}$. The remote terminal unit is installed to those refrigerated trucks which can monitor the inside temperature and humidity and get location from GPS module and send those information to owner's ERP system by wireless communication module. The ERP system analyses those trucks load data and running status and puts those trucks idle space data route information to cloud computing server which can put those information to e-commercial website. Other small customers can browse their required space with 
special route from the website and order those business by internet at any time. The order will turn back to truck owners and arrange their trucks to load those scatted refrigerated goods on their way.

This system architecture is as follows:

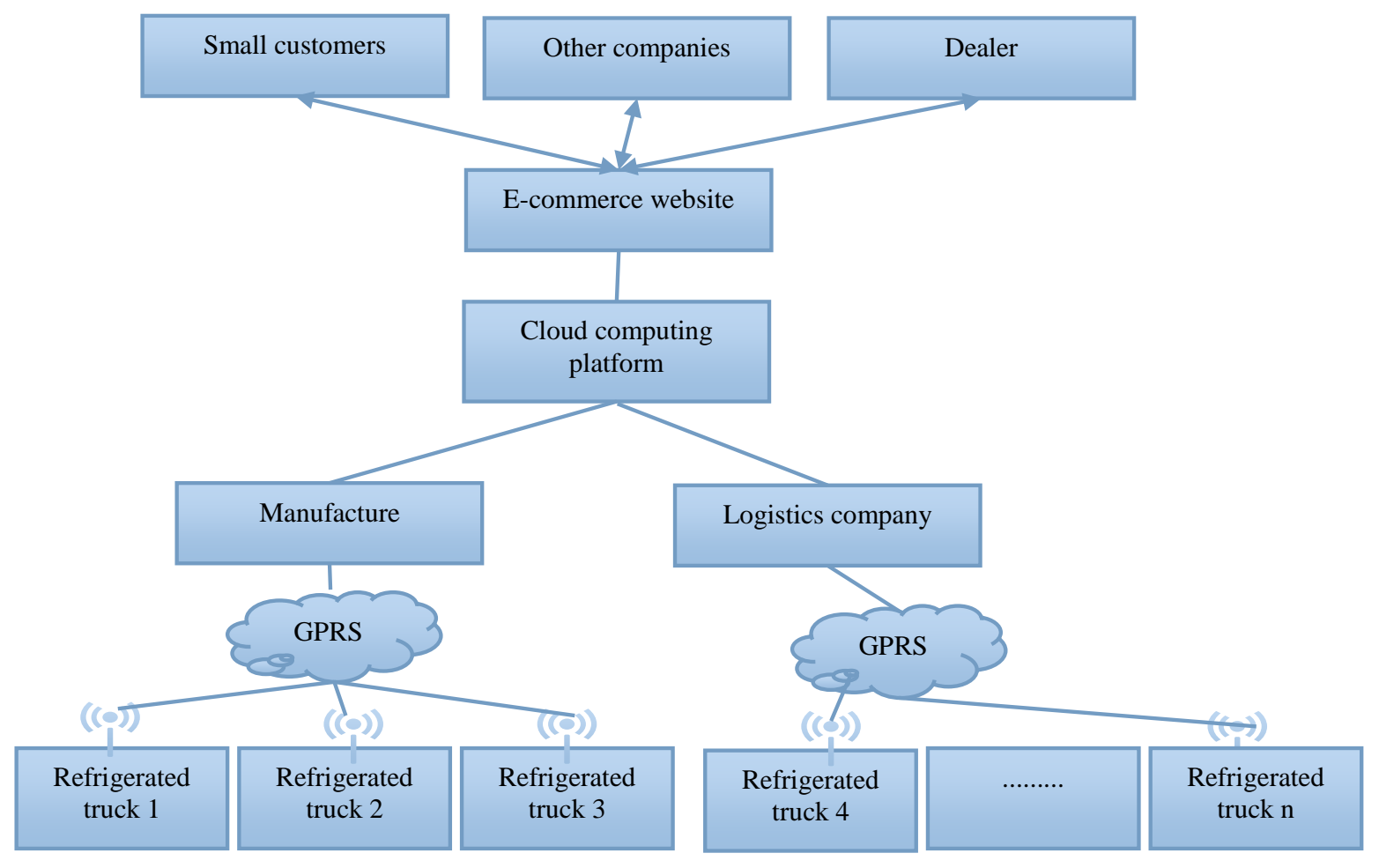

Fig 1.1 System architecture

\section{Hardware design}

The hardware refers the remote wireless terminal unit installed on those refrigerated trucks ${ }^{[4]}$. This unit is designed based on MSP430 as ARM 9 chip. The MSP430 connects ADT 7420 with IC ${ }^{2}$ to test the inside temperature, and connects GPS module with Rs232 to get the current location of this truck. When the necessary data is ready, the unit sends those data to the remote server through the GPRS module with RS232 port periodically. The schematic diagram of this unit is as follow Fig.2.

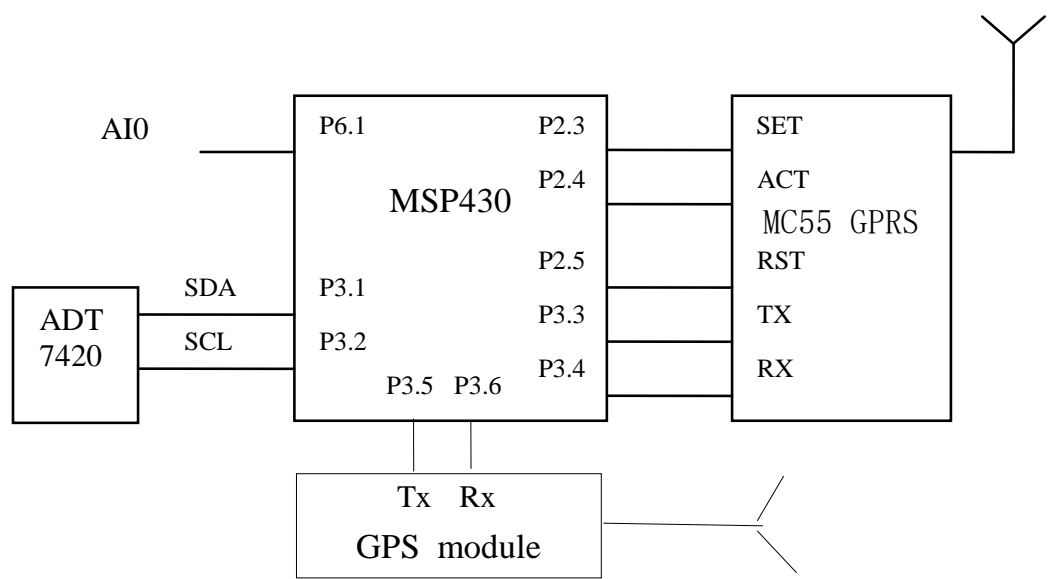

Fig .2. The schematic diagram of the remote wireless terminal unit 


\section{Software design}

The software of this system includes four parts as follows:

1. The server software receives the truck's running status data and current location data which always runs on the server of manufacture and Logistics company. This software function can be one part of those companies' ERP system. The communication between the server and those remote wireless terminal units is based on the MODBUS(TCP/IP) protocol widely used in data transmission between field devices and $\mathrm{PC}^{[5]}$. The server will receive the data frame every 15 minutes,. The data frame from the remote wireless terminal unit installed on the refrigerated truck includes the information of the real time location and inside temperature.

2. The second software function module analyzes those monitor data and those refrigerated trucks' load from work schedule and compute the idle space. If the idle space is valuable,this function module puts the data like the idle space, time, route to cloud computing platform running the e-commerce website. The data is selected from the local database server and sent to the e-commerce platform such as Ali cloud which is the biggest e-commerce platform in China. The protocol between the this function module and the cloud platform can be the Simple Object Access Protocol(SOAP $)^{[6]}$ which is designed to exchange structured and solidified information on WEB.

3. The e-commerce website provides a platform which connects information and potential customer with internet. The potential customers browse the information of idle cold-chain transport space , once they get the suitable truck, they will send a order to provider and pay the service quickly. This e-commerce website can be built on the Alibaba platform who can offer the main framework and open some resource to extend the necessary function module as customer's wash. The EC-Shop is a good development tool to complete this mission .

4. The owner of cold -chain transport system will arrange the scatted refrigerated goods' loading and unloading according to those orders. The order from the e-commerce system must be back to the truck owner's management system on time, and arranged immediately. The information is sent back to the remote wireless terminal at as soon as possibly in order to make sure that the customer's goods can be loaded on time.

The data flow is as follow:

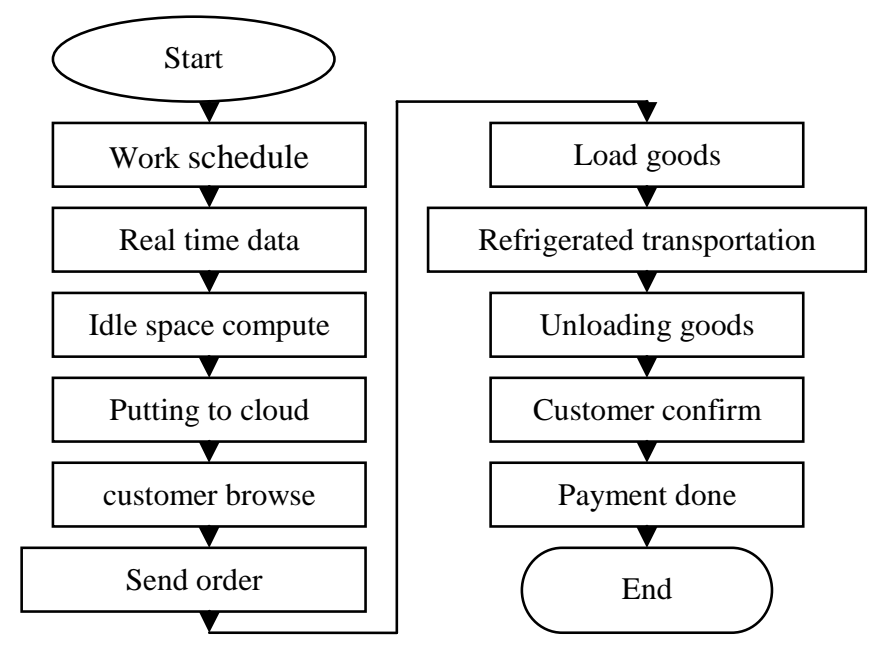

Fig.3. The data flow of system 
Here,with the help of the cloud computing and wireless network technology, this system can collect all the scattered idle space of refrigerated trucks and sell them to those potential customer without any refrigerated transport vehicle. By this way, those truck owners can running their refrigerated trucks with low price and high efficiency, on the other hand those small potential customers can transport their scattered refrigerated goods from one place to another place with a reasonable price and on time.

\section{Summary}

With the rapid development of China's economy,people's life level become more and more higher which causes the demand of refrigerated foods and products increasing rapidly. Those refrigerated goods can not be transport by regular Logistics company's truck, and the quality does not worth to rent a whole special refrigerated truck to transport from one place to another place. On the other hand, there are some waste in regular work schedule of big refrigerated products manufacture having refrigerated truck team. This system just build a connection between those idle refrigerated transport space and small customer which improves the cold-chain transport system greatly and reduce the cost of small customer.The e-commerce has many aspects of commercial including cold-chain transport system which can running more efficiently and lower cost. The cold chain transport industry will develop more and more fast with the the technology of wireless network and cloud computing. With the continuous improvement of people's quality of life requirements, more and more fresh goods will transport by cold chain transport system with reasonable price because of the new technology of internet.

\section{Acknowledgements}

This work was financially supported by the "High-end CNC machine tools and basic manufacturing equipment" major science and technology "vertical machining center made CNC systems supporting batch application engineering" (2014ZX04015011)

\section{References}

[1] http://baike.baidu.com/link?url=rvu0QNTrFnE4E176M7VqbWjRVIE484farfT-gmMa0VEGC7 mPEw6KCOaER_ve3tvLOCj8-wEywmYlHkKAaDskna

[2] Zhang Rangyong, Han Lingyan, Cheng guanghe, Zheng xiaoshi,in: the building envelope' $s$ thermal performance testing system based on infrared image and wireless networks ,advanced materials research vols 1030-1032.

[3] Geng Ma ,RangYong Zhang, in: Origin fresh beer remote delivery system based on cloud computing technology, ICMCE2014.

[4] Asadi G, Hosseini E. Cold supply chain management in processing of food and agricultural products.[J]. Scientific Papers, 2014.

[5] http://baike.baidu.com/link?url=HTSNIxJ47ymXG_lViXdgq6CfAf1qJJv_-ietlmMib7INX7E28 VKU6KA8ZQSuDX9pexhBIPsHbIzMkvvJrQwtWckrhzXNsFhAg-ih1v7kxzlfuCi73V0IsFE4n ZV2YvR0cCOL8IN5Pc47hK72oRrpiq

[6] http://baike.baidu.com/link?url=TSkyAE0SkH349eRZaqorQZTcO6kz_-DPD5U_Ns7Yl8h1qL 2kJBXRi9rylF5_Mqu7O2f514i5QS3xYfv5JCTpWLAmcqzCohxRrfp7bynlbs0IzOnH_Ty7eFV pRS-3M4eFGWwHVYc_LCS-LEPZEvlev_ 